\title{
Some Bond Incident Degree Indices of Cactus Graphs
}

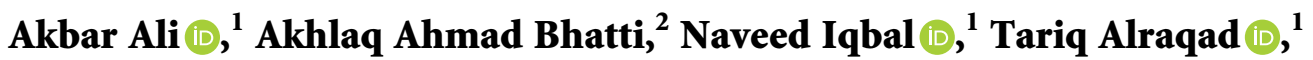 \\ Jaya Percival Mazorodze $\left(\mathbb{0},{ }^{3}\right.$ Hicham Saber, ${ }^{1}$ and Abdulaziz M. Alanazi $\mathbb{}^{4}{ }^{4}$ \\ ${ }^{1}$ Department of Mathematics, Faculty of Science, University of Ha'il, Ha'il, Saudi Arabia \\ ${ }^{2}$ Department of Sciences and Humanities, National University of Computer and Emerging Sciences, Lahore Campus, B-Block, \\ Faisal Town, Lahore, Pakistan \\ ${ }^{3}$ Department of Mathematics, University of Zimbabwe, Harare, Zimbabwe \\ ${ }^{4}$ School of Mathematics, University of Tabuk, Tabuk 71491, Saudi Arabia
}

Correspondence should be addressed to Jaya Percival Mazorodze; mazorodzejaya@gmail.com

Received 13 October 2021; Accepted 21 December 2021; Published 21 January 2022

Academic Editor: M. T. Rahim

Copyright (c) 2022 Akbar Ali et al. This is an open access article distributed under the Creative Commons Attribution License, which permits unrestricted use, distribution, and reproduction in any medium, provided the original work is properly cited.

A connected graph in which no edge lies on more than one cycle is called a cactus graph (also known as Husimi tree). A bond incident degree (BID) index of a graph $G$ is defined as $\sum_{u v \in E(G)} f\left(d_{G}(u), d_{G}(v)\right)$, where $d_{G}(w)$ denotes the degree of a vertex $w$ of $G, E(G)$ is the edge set of $G$, and $f$ is a real-valued symmetric function. This study involves extremal results of cactus graphs concerning the following type of the BID indices: $I_{f_{i}}(G)=\sum_{u v \in E(G)}\left[f_{i}\left(d_{G}(u)\right) / d_{G}(u)+f_{i}\left(d_{G}(v)\right) / d_{G}(v)\right]$, where $i \in\{1,2\}, f_{1}$ is a strictly convex function, and $f_{2}$ is a strictly concave function. More precisely, graphs attaining the minimum and maximum $I_{f}$ values are studied in the class of all cactus graphs with a given number of vertices and cycles. The obtained results cover several well-known indices including the general zeroth-order Randić index, multiplicative first and second Zagreb indices, and variable sum exdeg index.

\section{Introduction}

All the graphs considered in this study are connected. The notation and terminology that are used in this study but not defined here can be found in some standard graph-theoretical books $[6,7]$.

Graph invariants of the following form are known as the bond incident degree (BID) indices [5]:

$$
B I D(G)=\sum_{u v \in E(G)} f\left(d_{G}(u), d_{G}(v)\right),
$$

where $d_{G}(w)$ denotes the degree of a vertex $w \in V(G)$ of the graph $G, E(G)$ is the edge set of $G$, and $f$ is a real-valued symmetric function. In this study, we are concerned with the following type [2] of the BID indices:

$$
I_{f_{i}}(G)=\sum_{u v \in E(G)}\left[\frac{f_{i}\left(d_{G}(u)\right)}{d_{G}(u)}+\frac{f_{i}\left(d_{G}(v)\right)}{d_{G}(v)}\right]=\sum_{v \in V(G)} f_{i}\left(d_{G}(v)\right)
$$

where $i \in\{1,2\}, f_{1}$ is a strictly convex function, and $f_{2}$ is a strictly concave function.

A connected graph in which no edge lies on more than one cycle is called a cactus graph (also known as Husimi tree [9]). In the present study, we study the graphs attaining the minimum and maximum $I_{f_{i}}$ values from the class of all cactus graphs with a given number of vertices and cycles. Our main results cover the general zeroth-order Randic index ${ }^{0} R_{\alpha}$ [3], variable sum exdeg index $S E I_{a}$ [13], multiplicative first Zagreb index $\Pi_{1}$ [8], multiplicative second Zagreb index $\Pi_{2}[1,8,10]$, and sum lordeg index $S L[12,14]$, where the aforementioned indices for a graph $G$ are defined as follows:

$$
\begin{aligned}
{ }^{0} R_{\alpha}(G) & =\sum_{v \in V(G)}\left[d_{G}(v)\right]^{\alpha}, \\
S E I_{a}(G) & =\sum_{v \in V(G)} d_{G}(v) a^{d_{G}(v)},
\end{aligned}
$$




$$
\begin{aligned}
& \Pi_{1}(G)=\prod_{v \in V(G)}\left[d_{G}(v)\right]^{2}, \\
& \Pi_{2}(G)=\prod_{u v \in E(G)} d_{G}(u) d_{G}(v)=\prod_{v \in V(G)}\left[d_{G}(v)\right]^{d_{G}(v)}, \\
& S L(G)=\sum_{v \in V(G)} d_{G}(v) \sqrt{\ln \left[d_{G}(v)\right]} \\
& =\sum_{v \in V(G) ; d_{G}(v) \geq 2} d_{G}(v) \sqrt{\ln \left[d_{G}(v)\right]} .
\end{aligned}
$$

A graph in which every vertex has degree less than 5 is known as a chemical graph.

Although we cannot apply our main result on the Lanzhou index [15] for finding the extremal graphs from the class of all cactus graphs, we still are able to utilize one of our main results for finding the graphs having the minimum Lanzhou index among all chemical cactus graphs, where the Lanzhou index for a graph $G$ is defined as

$$
L z(G)=\sum_{v \in V(G)}\left(n-d_{G}(v)-1\right)\left[d_{G}(v)\right]^{2} .
$$

We end this section with the remark that the Lanzhou index is same [11] as the graph invariant ${ }^{0} R_{3}$.

\section{Main Results}

By an $n$-vertex graph, we mean a graph of order $n$. In a graph, a set of pairwise nonadjacent edges is called a matching. The elements of a matching are known as independent edges.

Theorem 1. The graph formed by adding $r$-independent edges in the n-vertex star $S_{n}$ (Figure 1) uniquely attains the maximum $I_{f_{1}}$ value and minimum $I_{f_{2}}$ value in the class of all $n$-vertex cactus graphs having $r$ cycles, where $n$ and $r$ are the fixed integers satisfying the inequalities $n \geq 2 r+1, n \geq 4$, and $r \geq 0$.

Proof. We prove the result for the graph invariant $I_{f_{1}}$. The result regarding the other invariant can be proved in a fully analogous way. Let $G$ be a graph having the maximum $I_{f}$ value in the given class of graphs. It is enough to show that $G$ has the maximum degree $n-1$. Contrarily, we assume that that the maximum degree of $G$ is at most $n-2$. Let $v \in V(G)$ be a vertex of maximum degree. Then, there exists at least one neighbor, say $w$ of $v$ which has at least one neighbor not adjacent to $v$. Let $w_{1}, w_{2}, \ldots, w_{k}$ be those neighbors of $w$ that are not adjacent to $v$. If $G^{\prime}$ is the graph formed by adding the edges $w_{1} v, w_{2} v, \ldots, w_{k} v$ in $G$ and removing the edges $w_{1} w, w_{2} w, \ldots, w_{k} w$ from $G$ (Figure 2), then we have

$$
\begin{aligned}
I_{f_{1}}(G)-I_{f_{1}}\left(G^{\prime}\right)= & f_{1}\left(d_{G}(v)\right)-f_{1}\left(d_{G}(v)+k\right) \\
& +f_{1}\left(d_{G}(w)\right)-f_{1}\left(d_{G}(w)-k\right) .
\end{aligned}
$$

Note that the cactus graphs $G$ and $G^{\prime}$ have the same number of cycles as well as order. By using Lagrange's mean

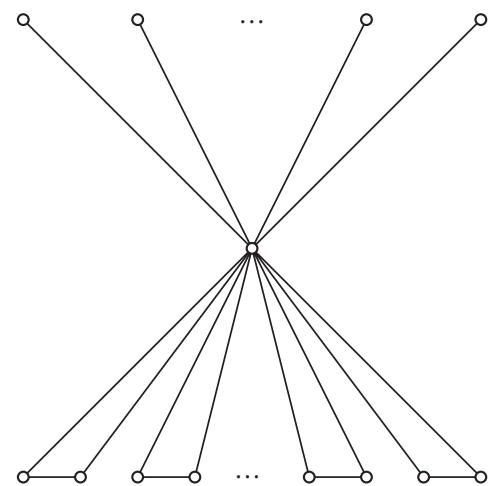

FIgure 1: The extremal graph mentioned in Theorem 1.
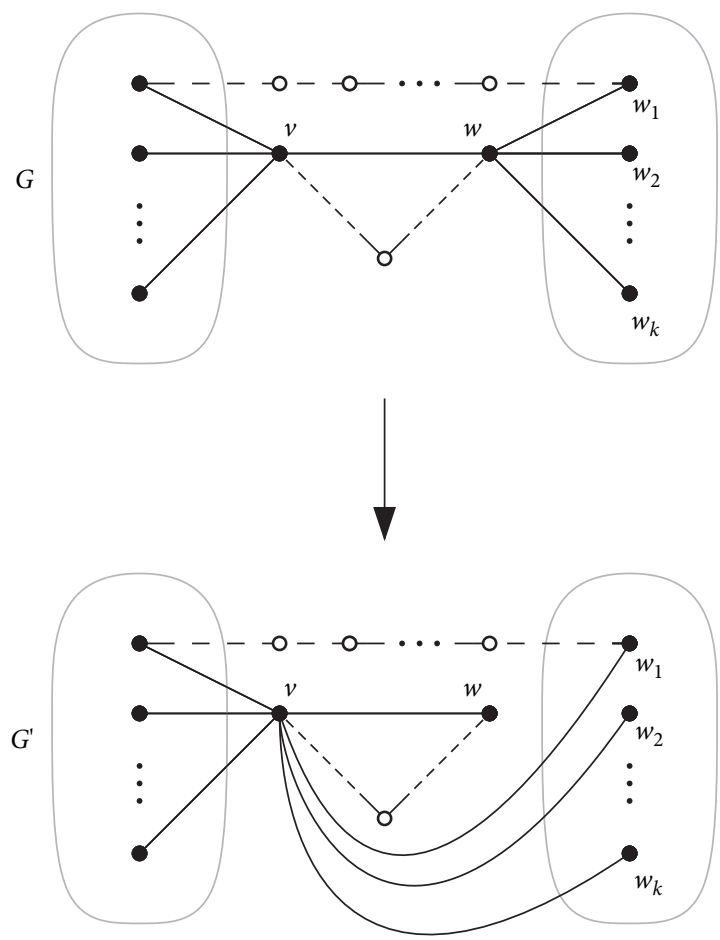

FIgURE 2: The graph transformation used in the proof of Theorem 1. The white vertices and doted edges may or may not exist provided that every edge lies on at most one cycle.

value theorem, we conclude that there exist real numbers $a_{1}$ and $a_{2}$, such that

$$
\begin{aligned}
a_{1} & \in\left(d_{G}(w)-k, d_{G}(w)\right), \\
a_{2} & \in\left(d_{G}(v), d_{G}(v)+k\right), \\
I_{f_{1}}(G)-I_{f_{1}}\left(G^{\prime}\right) & =k\left[f_{1}^{\prime}\left(a_{1}\right)-f_{1}^{\prime}\left(a_{2}\right)\right] .
\end{aligned}
$$

The fact $d_{G}(w) \leq d_{G}(v)$ implies that $a_{1}<a_{2}$, which further implies that the right hand side of equation (6) is negative because $f_{1}$ is a strictly convex function. Thus, one has $I_{f_{1}}(G)-I_{f_{1}}\left(G^{\prime}\right)<0$, a contradiction to the maximality of $I_{f_{1}}(G)$. 
Corollary 1. In the class of all n-vertex cactus graphs having $r$ cycles, the graph formed by adding $r$ independent edges in the $n$-vertex star $S_{n}$ uniquely attains the maximum general zeroth-order Randic index ${ }^{0} R_{\alpha}$ for $\alpha>1$ or $\alpha<0$, maximum variable sum exdeg index $S E I_{a}$ for $a>1$, maximum multiplicative second Zagreb index $\Pi_{2}$, maximum sum lordeg index $S L$, minimum general zeroth-order Randić index ${ }^{0} R_{\alpha}$ for $0<\alpha<1$, and minimum multiplicative first Zagreb index $\Pi_{1}$, where $n$ and $r$ are the fixed integers satisfying the inequalities $n \geq 2 r+1, n \geq 4$, and $r \geq 0$.

Proof. We observe that a graph $G$ attains its maximum $\Pi_{2}$ value or minimum $\Pi_{1}$ value in a class of graphs if and only if $G$ attains its maximum $\ln \Pi_{2}$ value or minimum $\ln \Pi_{1}$ value, respectively, in the considered class of graphs. We define $\phi_{1}(x)=x a^{x}$ with $a>1$ and $x \geq 1 ; \phi_{2}(x)=x^{\alpha}$ with $x \geq 1$ and $\alpha>1$ or $\alpha<0 ; \phi_{3}(x)=x \ln x$ with $x \geq 1 ; \phi_{4}(x)=x \sqrt{\ln x}$ with $x \geq 2 ; \phi_{5}(x)=2 \ln x$ with $x \geq 1$; and $\phi_{6}(x)=x^{\alpha}$ with $x \geq 1$ and $0<\alpha<1$. It can be easily verified that for each $i \in\{1,2,3,4\}, \phi_{i}$ is strictly convex and for each $j \in\{5,6\}, \phi_{j}$ is strictly concave. Thus, the required conclusion follows from Theorem 1.

A graph of order $n$ and size $m$ is called an $(n, m)$-graph.

Lemma 2 (see [4]). If $G$ attains the minimum $I_{f_{1}}$ value or maximum $I_{f_{2}}$ value among all connected $(n, m)$-graphs and $v \in V(G)$, then the minimum degree of $G$ is at least 2 , where $n$ and $m$ are the fixed integers satisfying the conditions $3 n \geq 2 m$, $n \geq 4$, and $m \geq n$.

The next result is a direct consequence of Lemma 2 .

Corollary 3. If $G$ is a graph attaining the minimum $I_{f_{1}}$ value or maximum $I_{f_{2}}$ value in the class of all $n$-vertex cactus graphs having $r$ cycles, then the minimum degree of $G$ is 2 , where $n$ and $r$ are the fixed integers satisfying the inequalities $n \geq 2 r+1, n \geq 6$, and $r \geq 2$.

Denote by $N_{G}(v)$ the set of neighbors of a vertex $v \in V(G)$ of a graph $G$.

Theorem 2. If $G$ is a graph attaining the minimum $I_{f_{1}}$ value or maximum $I_{f_{2}}$ value in the class of all $n$-vertex cactus graphs having $r$ cycles and $v \in V(G)$, then the minimum degree of $G$ is 2 and

$$
d_{G}(v) \leq \begin{cases}4, & \text { if } v \text { lies on some cycle, } \\ 3, & \text { otherwise }\end{cases}
$$

where $n$ and $r$ are the fixed integers satisfying the inequalities $n \geq 2 r+1, n \geq 6$, and $r \geq 2$.

Proof. We prove the result for the graph invariant $I_{f_{1}}$. The result regarding the other invariant can be proved in a fully analogous way. Let $G$ be a graph having the minimum $I_{f_{1}}$ value in the given class of graphs.

From Corollary 3, it follows that the minimum degree of $G$ is 2 . Next, we prove that

$$
d_{G}(v) \leq \begin{cases}4, & \text { if } v \text { lies on some cycle } \\ 3, & \text { otherwise }\end{cases}
$$

First, assume that $v$ lies one some cycle $C$ of $G$. Suppose to the contrary that $d_{G}(v) \geq 5$. Let $N_{G}(v) \backslash V(C)$ : = $\left\{v_{1}, v_{2}, \ldots, v_{r}\right\}$, where $V(C)$ denotes the set of vertices of the cycle $C$. For $i=1,2, \ldots, r$, denote by $M_{i}$ the component of the graph $G-\{v\}$ containing the vertex $v_{i}$. It is claimed that no more than two vertices of $N_{G}(v) \backslash V(C)$ lie on the same component of the graph $G-\{v\}$; if $v_{k}$, $v_{l}$, and $v_{m}$ lie on the same component of the graph $G-\{v\}$, then the vertices $v_{k}, v_{l}$, $v_{m}$, and $v$ lie on a cycle whose each edge belongs to more than one cycle of $G$, which contradicts the definition of $G$.

Case 1. There exists at least one $i$, such that the component $M_{i}$ contains a unique vertex of $N_{G}(v) \backslash V(C)$.

Suppose, without loss of generality, that the component $M_{1}$ contains none of $v_{2}, v_{3}, \ldots, v_{r}$. We note that there exists at least one component $M_{j}$ with $j \geq 2$, such that $M_{j}$ contains at least one vertex $u \in V(G)$ satisfying $d_{G}(u)=2$. Certainly, both the graphs $G$ and $G^{\prime} \cong G-\left\{v v_{1}\right\}+\left\{u v_{1}\right\}$ have the same number of cycles and vertices. On the other hand, we have

$$
\begin{aligned}
I_{f_{1}}(G)-I_{f_{1}}\left(G^{\prime}\right)= & f_{1}\left(d_{G}(v)\right)-f_{1}\left(d_{G}(v)-1\right) \\
& +f_{1}\left(d_{G}(u)\right)-f_{1}\left(d_{G}(u)+1\right) \\
= & f_{1}\left(d_{G}(v)\right)-f_{1}\left(d_{G}(v)-1\right) \\
& -\left[f_{1}(3)-f_{1}(2)\right] .
\end{aligned}
$$

By using Lagrange's mean value theorem, we conclude that there exist real numbers $a_{1}$ and $a_{2}$, such that

$$
\begin{aligned}
a_{1} & \in(2,3), \\
a_{2} & \in\left(d_{G}(v)-1, d_{G}(v)\right), \\
I_{f_{1}}(G)-I_{f_{1}}\left(G^{\prime}\right) & =f_{1}^{\prime}\left(a_{2}\right)-f_{1}^{\prime}\left(a_{1}\right) .
\end{aligned}
$$

The assumption $d_{G}(v) \geq 5$ implies that $a_{1}<a_{2}$, which further implies that the right hand side of equation (2) is positive because $f_{1}$ is a strictly convex function. Thus, one has $I_{f_{1}}(G)-I_{f_{1}}\left(G^{\prime}\right)>0$, a contradiction to the minimality of $I_{f_{1}}(G)$.

Case 2. For each $i \in\{1,2, \ldots, r\}$, exactly two vertices of the set $N_{G}(v) \backslash V(C)$ lie on the component $M_{i}$.

Suppose, without loss of generality, that $M_{1}=M_{2}$. It is clear that $M_{1} \neq M_{j}$ for each $j \in\{3, \ldots, r\}$, and there exists at least one component $M_{j}$ with $j \geq 3$, such that $M_{j}$ contains at least one vertex $w \in V(G)$ satisfying $d_{G}(w)=2$. It is obvious that both the graphs $G$ and $G^{\prime \prime} \cong G-\left\{v v_{1}, v v_{2}\right\}+\left\{w v_{1}, w v_{2}\right\}$ have the same number of cycles and vertices. On the other hand, we have

$$
\begin{aligned}
I_{f_{1}}(G)-I_{f_{1}}\left(G^{\prime \prime}\right)= & f_{1}\left(d_{G}(v)\right)-f_{1}\left(d_{G}(v)-2\right) \\
& +f_{1}\left(d_{G}(w)\right)-f_{1}\left(d_{G}(w)+2\right) \\
= & f_{1}\left(d_{G}(v)\right)-f_{1}\left(d_{G}(v)-2\right) \\
& -\left[f_{1}(4)-f_{1}(2)\right] .
\end{aligned}
$$


By using Lagrange's mean value theorem, we conclude that there exist real numbers $a_{3}$ and $a_{4}$, such that

$$
\begin{aligned}
a_{3} & \in(2,4), \\
a_{4} & \in\left(d_{G}(v)-2, d_{G}(v)\right), \\
I_{f_{1}}(G)-I_{f_{1}}\left(G^{\prime \prime}\right) & =2\left[f_{1}^{\prime}\left(a_{4}\right)-f_{1}^{\prime}\left(a_{3}\right)\right] .
\end{aligned}
$$

We note, for the present case, that the degree of $v$ is at least 6 , which implies that $a_{3}<a_{4}$, which further implies that the right hand side of (12) is positive because $f_{1}$ is a strictly convex function. Thus, one has $I_{f_{1}}(G)-I_{f_{1}}\left(G^{\prime \prime}\right)>0$, a contradiction to the minimality of $I_{f_{1}}(G)$.

Thus, $d_{G}(v) \leq 4$ when $v$ lies on some cycle of $G$.

It is still left to prove that $d_{G}(v) \leq 3$ when $v$ does not belong to any cycle of $G$. Suppose to the contrary that $d_{G}(v)=r \geq 4$ and that $v$ does not belong to any cycle of $G$. As before, we take $N_{G}(v)=\left\{v_{1}, v_{2}, \ldots, v_{r}\right\}$, and for $i \in\{1,2, \ldots, r\}$, we denote by $M_{i}$ the component of the graph $G-v$ containing the vertex $v_{i}$. We observe that $M_{i} \neq M_{j}$ whenever $i \neq j$; if the components $M_{i}$ and $M_{j}$ are the same for some $i \neq j$, then the path from $v_{i}$ to $v_{j}$ in $G-\{v\}$ together with the path $v_{i} v v_{j}$ yields a cycle in $G$ containing $v$, which is a contradiction. We note that there exists at least one component $M_{j}$ with $j \geq 2$, such that $M_{j}$ contains at least one vertex $x \in V(G)$ satisfying $d_{G}(x)=2$. It is obvious that both the graphs $G$ and $G^{\prime \prime \prime} \cong G-\left\{v_{1} v\right\}+\left\{v_{1} x\right\}$ have the same number of cycles and vertices. On the other hand, we have

$$
\begin{aligned}
I_{f_{1}}(G)-I_{f_{1}}\left(G^{\prime \prime}\right)= & f_{1}\left(d_{G}(v)\right)-f_{1}\left(d_{G}(v)-1\right) \\
& +f_{1}\left(d_{G}(x)\right)-f_{1}\left(d_{G}(x)+1\right) \\
= & f_{1}\left(d_{G}(v)\right)-f_{1}\left(d_{G}(v)-1\right) \\
& -\left[f_{1}(3)-f_{1}(2)\right] .
\end{aligned}
$$

By using Lagrange's mean value theorem, we conclude that there exist real numbers $a_{5}$ and $a_{6}$, such that

$$
\begin{aligned}
a_{5} & \in(2,3), \\
a_{6} & \in\left(d_{G}(v)-1, d_{G}(v)\right), \\
I_{f_{1}}(G)-I_{f_{1}}\left(G^{\prime \prime}\right) & =f_{1}^{\prime}\left(a_{6}\right)-f_{1}^{\prime}\left(a_{5}\right) .
\end{aligned}
$$

The assumption $d_{G}(v) \geq 4$ implies that $a_{3}<a_{4}$, which further implies that the right hand side of equation (4) is positive because $f_{1}$ is a strictly convex function. Thus, one has $I_{f_{1}}(G)-I_{f_{1}}\left(G^{\prime \prime}\right)>0$, a contradiction to the minimality of $I_{f_{1}}(G)$. Thus, $d_{G}(v) \leq 3$ when $v$ does not belong to any cycle of $G$.

Corollary 4. If $G$ is a graph attaining the minimum general zeroth-order Randić index ${ }^{0} R_{\alpha}$ for $\alpha>1$ or $\alpha<0$, minimum variable sum exdeg index $S E I_{a}$ for $a>1$, minimum multiplicative second Zagreb index $\Pi_{2}$, minimum sum lordeg index SL, maximum general zeroth-order Randić index ${ }^{0} R_{\alpha}$ for $0<\alpha<1$, and maximum multiplicative first Zagreb index $\Pi_{1}$, in the class of all $n$-vertex cactus graphs having $r$ cycles and $v \in V(G)$, then the minimum degree of $G$ is 2 and

$$
d_{G}(v) \leq \begin{cases}4, & \text { if } v \text { lies on some cycle } \\ 3, & \text { otherwise }\end{cases}
$$

where $n$ and $r$ are the fixed integers satisfying the inequalities $n \geq 2 r+1, n \geq 6$, and $r \geq 2$.

Proof. We observe that a graph $G$ attains its minimum $\Pi_{2}$ value or maximum $\Pi_{1}$ value in a class of graphs if and only if $G$ attains its minimum $\ln \Pi_{2}$ value or maximum $\ln \Pi_{1}$ value, respectively, in the considered class of graphs. We define $\phi_{1}(x)=x a^{x}$ with $a>1$ and $x \geq 1 ; \phi_{2}(x)=x^{\alpha}$ with $x \geq 1$ and $\alpha>1$ or $\alpha<0 ; \phi_{3}(x)=x \ln x$ with $x \geq 1 ; \phi_{4}(x)=x \sqrt{\ln x}$ with $x \geq 2 ; \phi_{5}(x)=2 \ln x$ with $x \geq 1$; and $\phi_{6}(x)=x^{\alpha}$ with $x \geq 1$ and $0<\alpha<1$. It can be easily verified that for each $i \in\{1,2,3,4\}, \phi_{i}$ is strictly convex, and for each $j \in\{5,6\}, \phi_{j}$ is strictly concave. Thus, the required conclusion follows from Theorem 2.

We observe that the function $\psi(x)=(n-1-x) x^{2}$ is strictly convex for $x<(n-1) / 3$. Thus, we have the next corollary regarding the Lanzhou index.

Corollary 5. If $G$ is a graph attaining the minimum Lanzhou index in the class of all n-vertex chemical cactus graphs having $r$ cycles and $v \in V(G)$, then the minimum degree of $G$ is 2 and

$$
d_{G}(v) \leq \begin{cases}4, & \text { if } v \text { lies on some cycle, } \\ 3, & \text { otherwise, }\end{cases}
$$

where $n$ and $r$ are the fixed integers satisfying the inequalities $n \geq 2 r+1, n \geq 6$, and $r \geq 2$.

\section{Data Availability}

The data used to support the findings of this study are available from the corresponding author upon request.

\section{Conflicts of Interest}

The authors declare that they have no conflicts of interest.

\section{Acknowledgments}

This research was funded by Scientific Research Deanship at University of Ha'il, Saudi Arabia, through project number RG-20 031.

\section{References}

[1] I. R. Abdolhosseinzadeh, F. Rahbarnia, and M. Tavakoli, "Some indices of edge corona of two graphs," Applied Mathematics E-Notes, vol. 18, pp. 13-24, 2018.

[2] A. Ali, Some vertex-degree-based topological indices of graphs, $\mathrm{PhD}$ thesis, National University of Computer and Emerging Sciences, Lahore, Pakistan, 2016.

[3] A. Ali, I. Gutman, E. Milovanović, and I. Milovanović, "Sum of powers of the degrees of graphs: extremal results and bounds," MATCH Communications in Mathematical and in Computer Chemistry, vol. 80, pp. 5-84, 2018.

[4] A. Ali, I. Gutman, I. Gutman, H. Saber, and A. Alanazi, "On bond incident degree indices of (n,m) -graphs," Match 
Communications in Mathematical and in Computer Chemistry, vol. 87, no. 1, pp. 89-96, 2022.

[5] A. Ali, Z. Raza, and A. A. Bhatti, "Bond incident degree (BID) indices of polyomino chains: a unified approach," Applied Mathematics and Computation, vol. 287-288, pp. 28-37, 2016.

[6] J. A. Bondy and U. S. R. Murty, Graph Theory, Springer, London, UK, 2008.

[7] G. Chartrand, L. Lesniak, and P. Zhang, Graphs \& Digraphs, CRC Press, Boca Raton, FL, USA, 2016.

[8] I. Gutman, "Multiplicative Zagreb indices of trees," Bulletin of International Mathematical Virtual Institute, vol. 1, pp. 13-19, 2011.

[9] F. Harary and G. E. Uhlenbeck, "On the number of Husimi trees: I," Proceedings of the National Academy of Sciences, vol. 39, no. 4, pp. 315-322, 1953.

[10] A. Iranmanesh, M. Hosseinzadeh, and I. Gutman, "On multiplicative Zagreb indices of graphs," Iranian Journal of Mathematical Chemistry, vol. 3, pp. 145-154, 2012.

[11] I. Milovanović, M. Matejić, and E. Milovanović, "A note on the general zeroth-order Randić coindex of graphs," Contributions to Mathematics, vol. 1, pp. 17-21, 2020.

[12] I. Tomescu, "Properties of connected -graphs extremal relatively to vertex degree function index for convex functions," MATCH Communications in Mathematical and in Computer Chemistry, vol. 85, pp. 285-294, 2021.

[13] B. Vukičević, "Bond additive modeling 4. QSPR and QSAR studies of the variable Adriatic indices," Croatica Chemica Acta, vol. 84, pp. 87-91, 2011.

[14] D. Vukičević and M. Gašperov, "Bond additive modeling 1. Adriatic indices," Croatica Chemica Acta, vol. 83, pp. 43-260, 2010.

[15] D. Vukičević, Q. Li, J. Sedlar, and T. Došlić, "Lanzhou index," MATCH Communications in Mathematical and in Computer Chemistry, vol. 80, pp. 863-876, 2018. 